Supporting Information

\title{
Wavelength Dependent Charge Carrier Dynamics for Single Pixel Color Sensing using Graded Perovskite Structures
}

\author{
N. Ganesh ${ }^{1}$, Ravichandran Shivanna ${ }^{2 *}$, Richard. H. Friend ${ }^{2 *}$ and K.S. Narayan ${ }^{1,3 *}$ \\ ${ }^{1}$ Chemistry and Physics of Materials Unit, Jawaharlal Nehru Center for Advanced Scientific
}

Research, Jakkur, Bengaluru-560064, India

Email: narayan@jncasr.ac.in

${ }^{2}$ Cavendish Laboratory, University of Cambridge, Cambridge CB3 0HE, United Kingdom

Email: rs919@cam.ac.uk; rhf10@cam.ac.uk

${ }^{3}$ School of Advanced Materials (SAMat), Jawaharlal Nehru Center for Advanced Scientific

Research, Jakkur, Bengaluru-560064, India

\section{List of contents:}

1. Absorption spectra of MAI and FAI based graded bandgap perovskite

2. Two-Photon confocal microscopy on graded bandgap perovskite film.

3. I-V and IPCE of Asymmetric device

4. Transient photocurrent showing negligible delay for different wavelength of illumination

5. Transient photocurrent profile in a symmetric hole-only device upon variation of illumination intensity.

6. Normalized photocurrent profiles and slopes for wavelength identification

7. Linear interpolation of $\mathrm{I}_{\mathrm{ph}}(\mathrm{t})$ for different $\lambda$ from experimentally acquired features

8. Difference between spectrally pure and reconstructed green.

9. Steady state light pulsing measurements on control symmetric devices

10. Spectral responsivity of symmetric graded bandgap devices 
11. Off-cycle photocurrent decay profiles for different intensities of light illumination.

12. Circuit analysis of the steady state photocurrent features.

13. Effect of capacitance variation on off-cycle $\mathrm{I}_{\mathrm{ph}}$ decay features.

14. Steady-state light pulsing in symmetric hole-only devices and the equivalent circuit

15. Fitting of capacitive decay equation to transient-OFF features in asymmetric devices

16. Table of decay time parameters in off-cycle features

17. Experimental Section 
1. Absorption spectra of Methylammonium Iodide (MAI) and Formamidium Iodide (FAI) based graded bandgap perovskite.

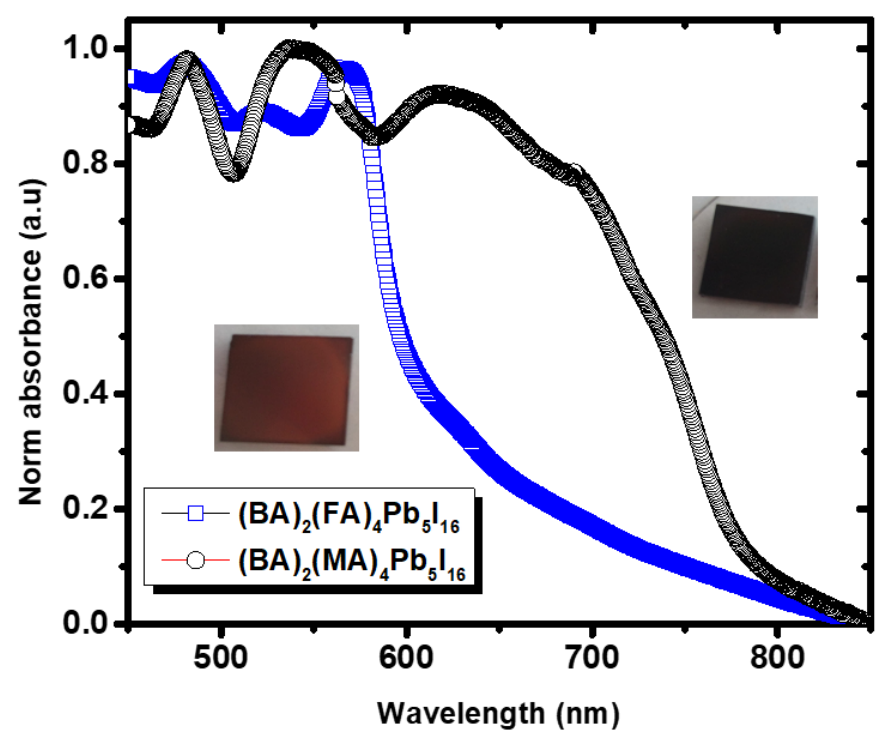

Figure S1. Normalized absorption spectra of mixed 2D/3D graded bandgap perovskite films.

2. Two-Photon confocal microscopy on graded bandgap perovskite film.
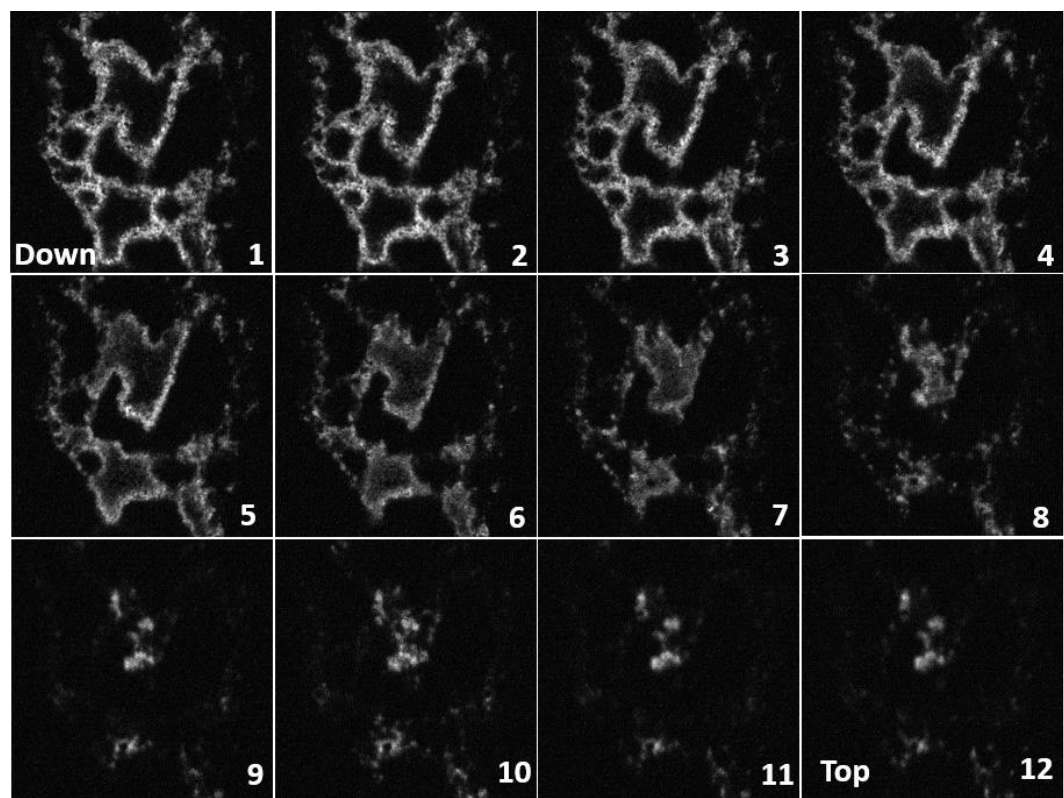

Figure S2. z-stack of 2-photon confocal on graded bandgap perovskite using a pulsed excitation $(\sim 100 \mathrm{fs})$ at $800 \mathrm{~nm}$ fundamental. The emission was collected through a GFP filter $(\sim 520 \mathrm{~nm}$, bandwidth $\sim 70 \mathrm{~nm}$ ). The trend of emission collected across the thickness of the film reveal features with large domains of $2 \mathrm{D}$ perovskite closer to the substrate which reduces to the top of the film. 
3. I-V and IPCE of Asymmetric device
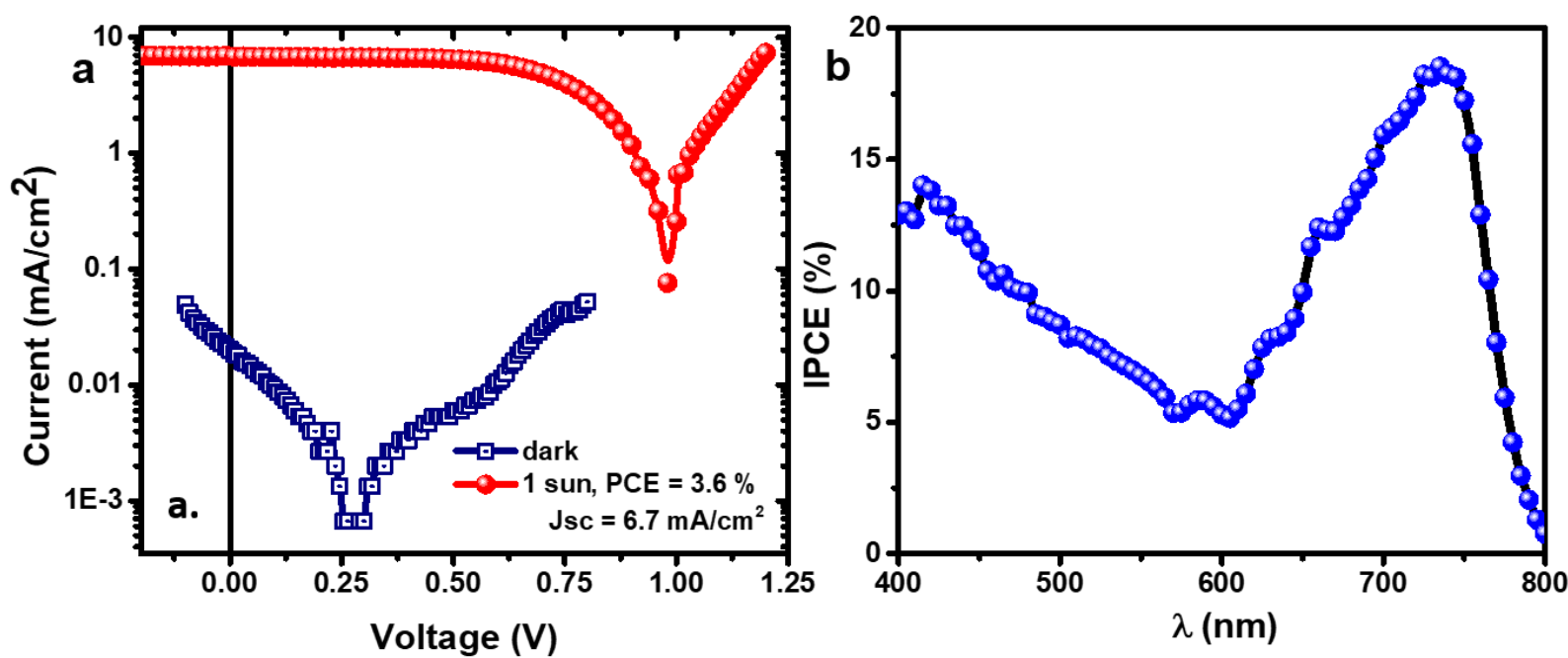

Figure S3. a) I(V) scan of asymmetric device with graded bandgap perovskite as the active layer done in dark and under 1sun which show PCE close to 4\%. b) IPCE data on asymmetric devices across the UV-Vis spectrum.

4. Initial part of the Transient photocurrent showing negligible delay for different wavelength of illumination

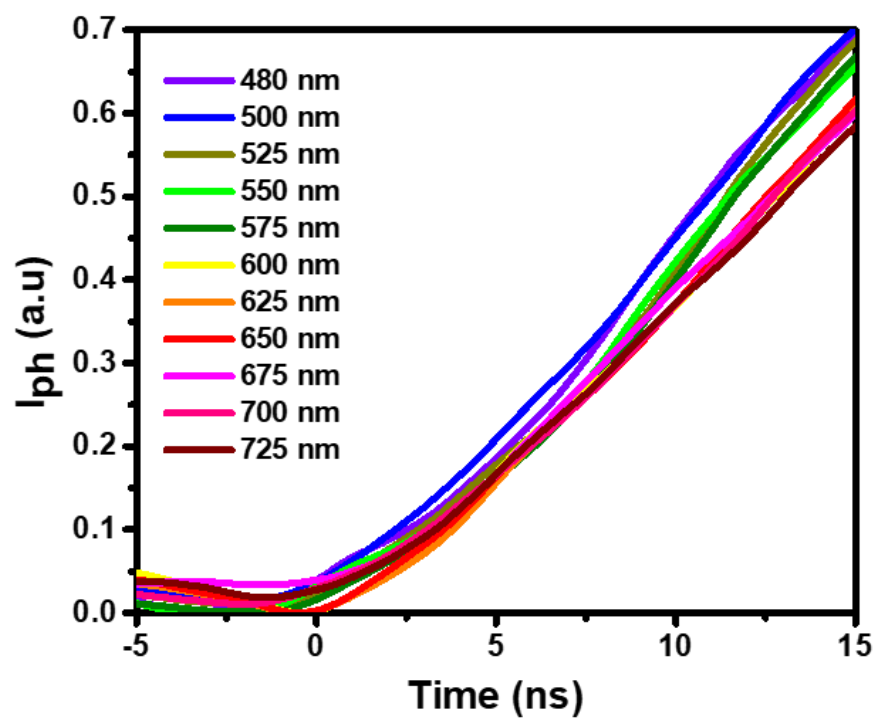

Figure S4. Initial part of ultrafast transient photocurrent profiles indicating negligible delay in the light illumination at different wavelengths. 
5. Transient photocurrent profile in a symmetric hole-only device upon variation of illumination intensity.
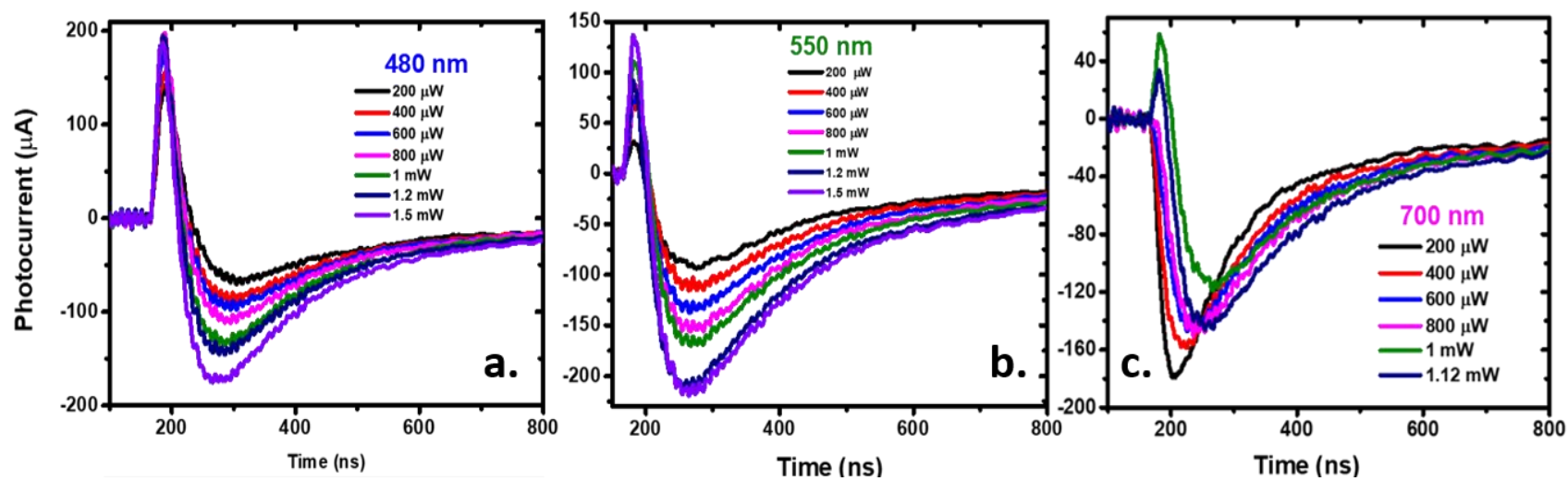

Figure S5. Ultrafast photocurrent on symmetric devices indicating intensity dependent photocurrent profile with illumination at a) $480 \mathrm{~nm}$ b) $550 \mathrm{~nm}$ and c) $700 \mathrm{~nm}$.

6. Normalized photocurrent profiles and slopes for wavelength identification
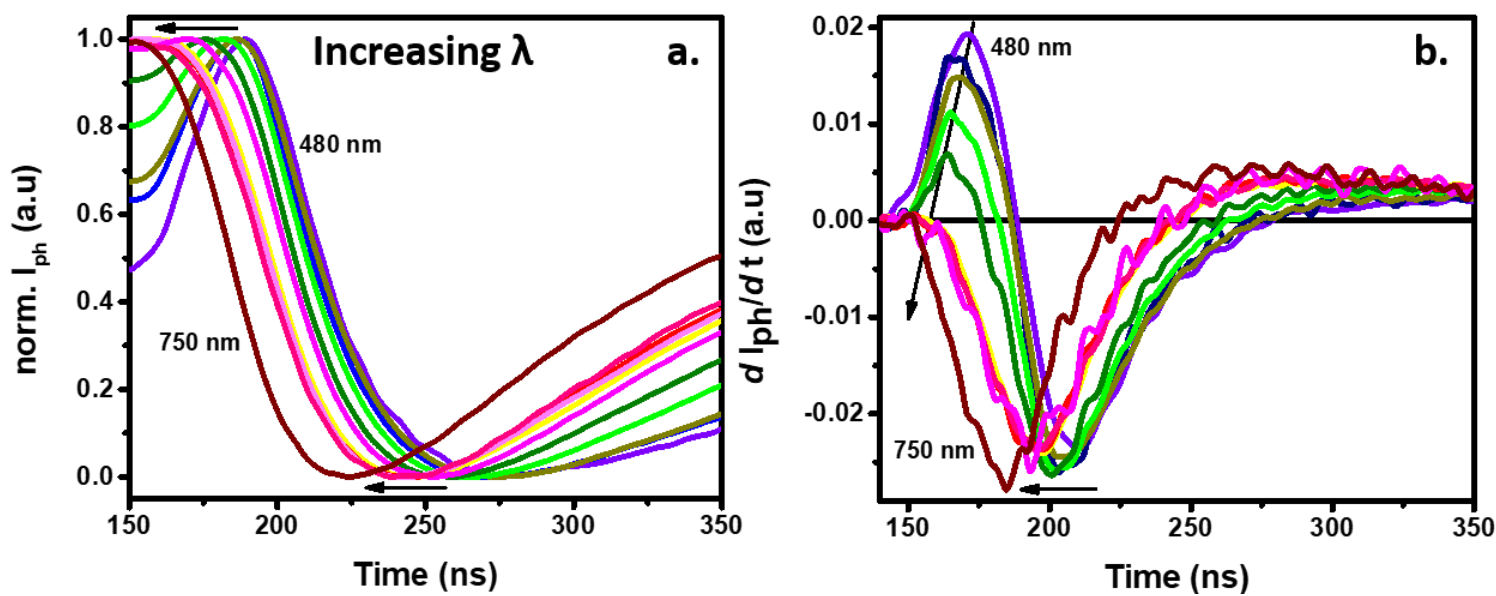

Figure S6. a) Photocurrent features (Figure 3c) smoothed and normalized in the range $[0,1]$ shows that the photocurrent minima appears earlier in case of longer wavelengths. b) Slopes of photocurrent features showing increased rate of charge extraction for lower wavelengths in the positive transients. The early arrival of $\mathrm{I}_{\mathrm{ph}}$ minima is also reflected at the zero-crossing point. 
7. Linear interpolation of $\mathrm{I}_{\mathrm{ph}}(\mathrm{t})$ for different $\lambda$ from experimentally acquired features
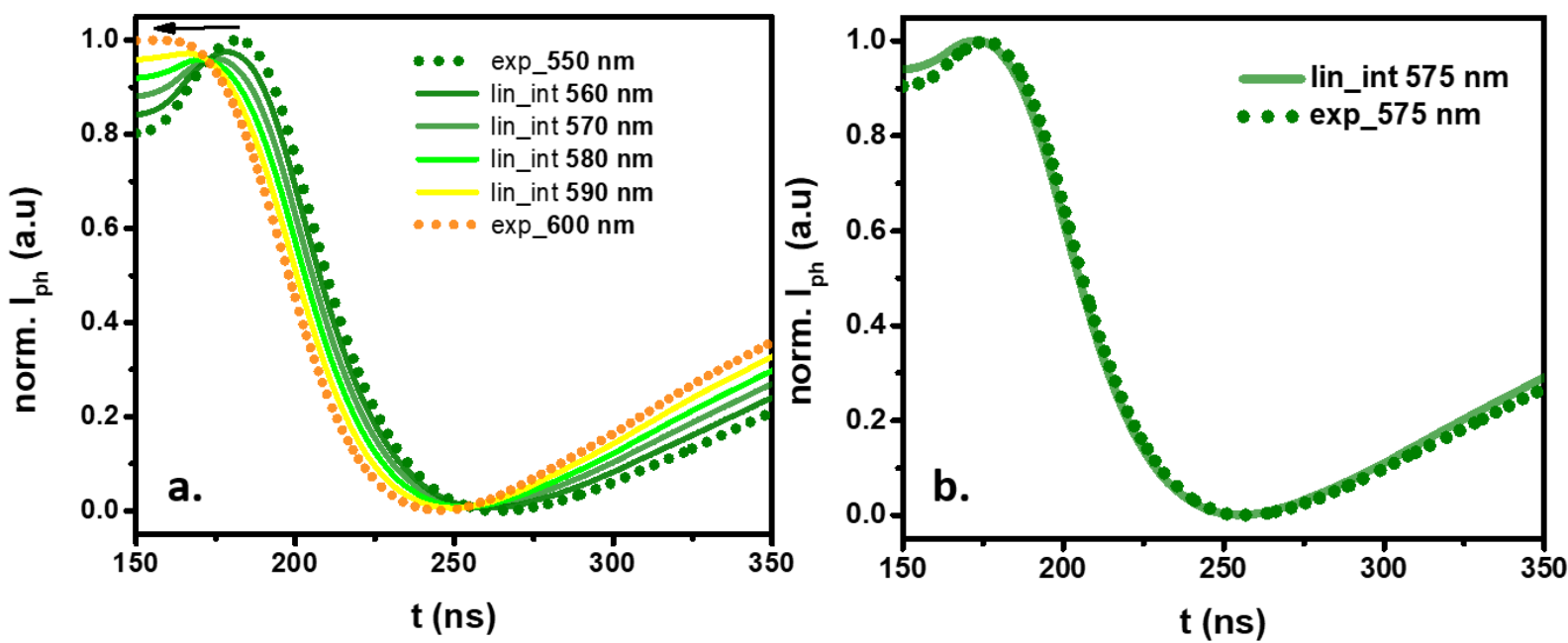

Figure S7. a) $I_{p h}(t)$ features (normalized in $[0,1]$ ) determined by interpolation for $\lambda$ in the interval between two experimentally acquired sets of data. $\mathrm{I}_{\mathrm{ph}}(\mathrm{t})$ were acquired at $550 \mathrm{~nm}$ and $600 \mathrm{~nm}$. The intermediate features were obtained on linear interpolation. b) Plot shows good fit between the $\mathrm{I}_{\mathrm{ph}}(\mathrm{t})$ for $575 \mathrm{~nm}$ excitation and the feature obtained on interpolation.

8. Difference between spectrally pure and reconstructed green.

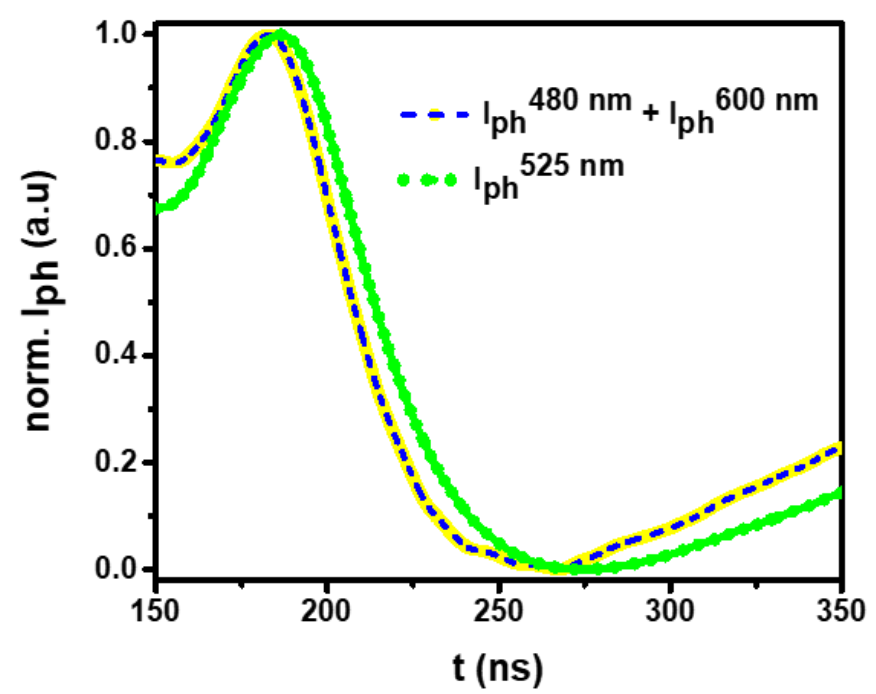

Figure S8. Plots shows the difference between a $\mathrm{I}_{\mathrm{ph}}(\mathrm{t})$ upon pure green $(525 \mathrm{~nm})$ excitation and a constructed green response obtained on summation of blue and yellow illumination $\mathrm{I}_{\mathrm{ph}}(\mathrm{t})$ features obtained from Figure 3a. Features were normalized to $[0,1]$ after summation. 
9. Steady state light pulsing measurements on control symmetric devices

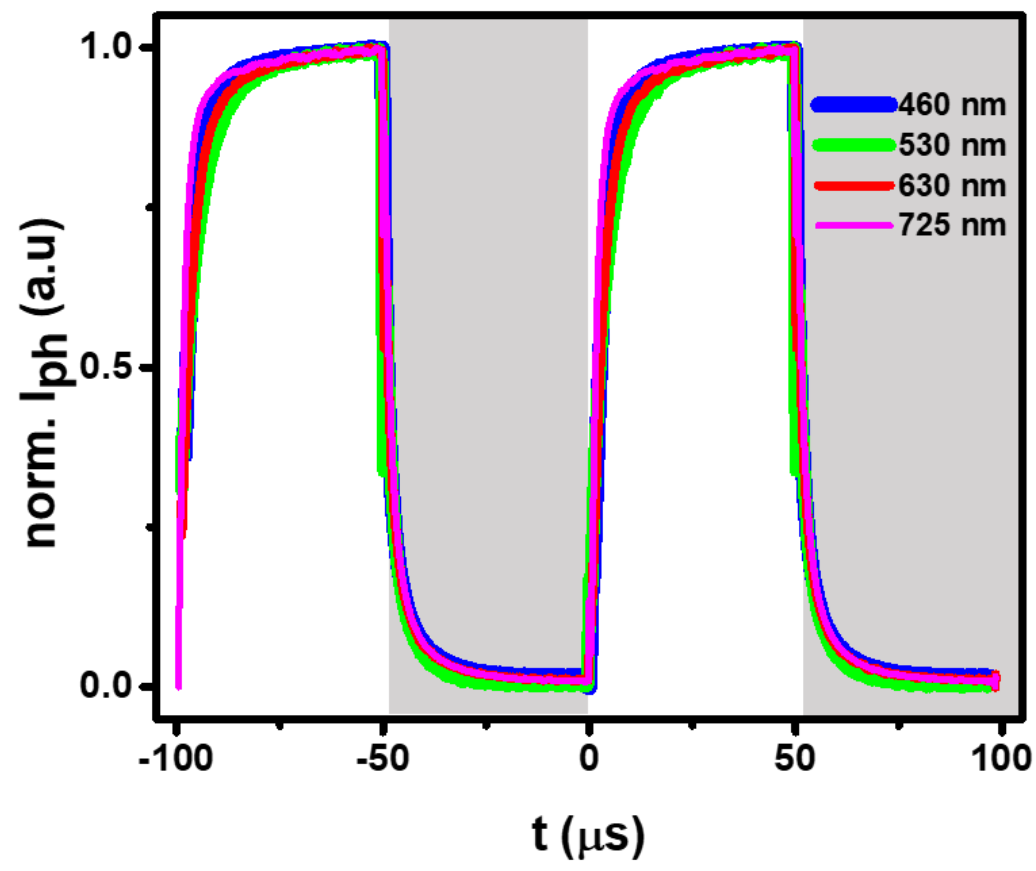

Figure S9. Steady state light pulsing measurement of control (3-D) symmetric device in shortcircuit mode, using a preamplifier and digital signal oscilloscope. The device configuration in this case was: ITO/PEDOT-PSS/3D-MAPbI $3 / \mathrm{P} 3 \mathrm{HT} / \mathrm{Ag}$. The bright regions represent the ON part and the shaded regions represent the OFF part of the light pulse.

10. Spectral Responsivity of symmetric graded bandgap detector.

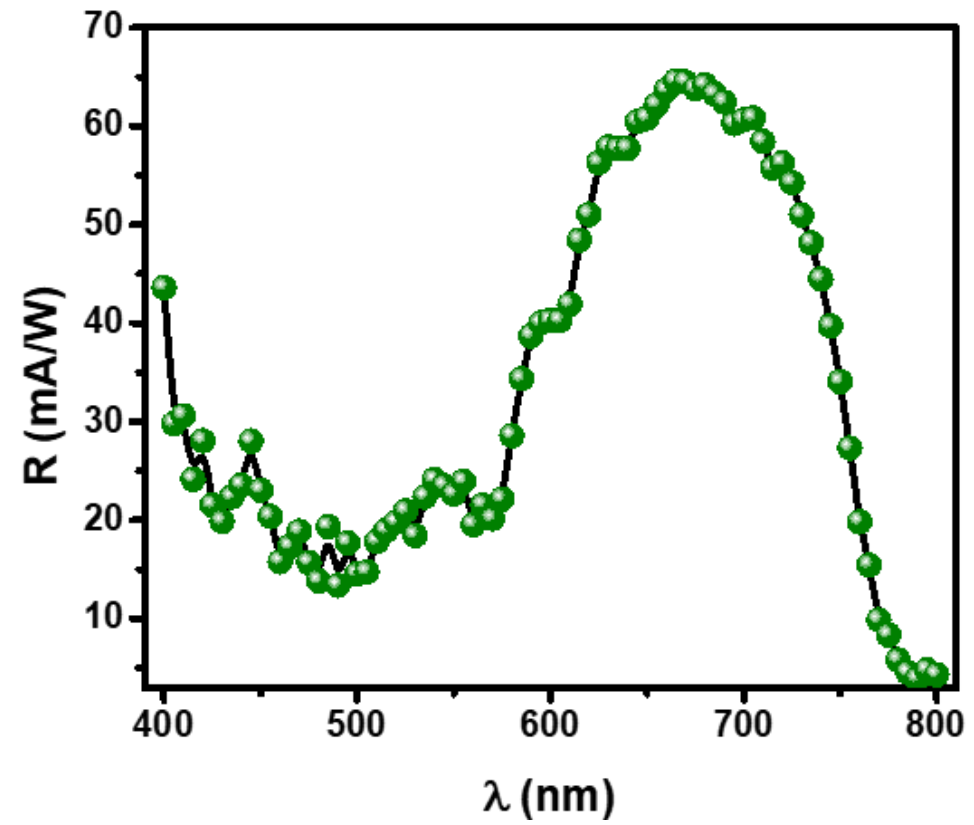

Figure S10. Spectral Responsivity in a graded bandgap symmetric device (0 V bias, Shortcircuit mode) 
11. Off-cycle photocurrent decay profiles for different intensities of light illumination.
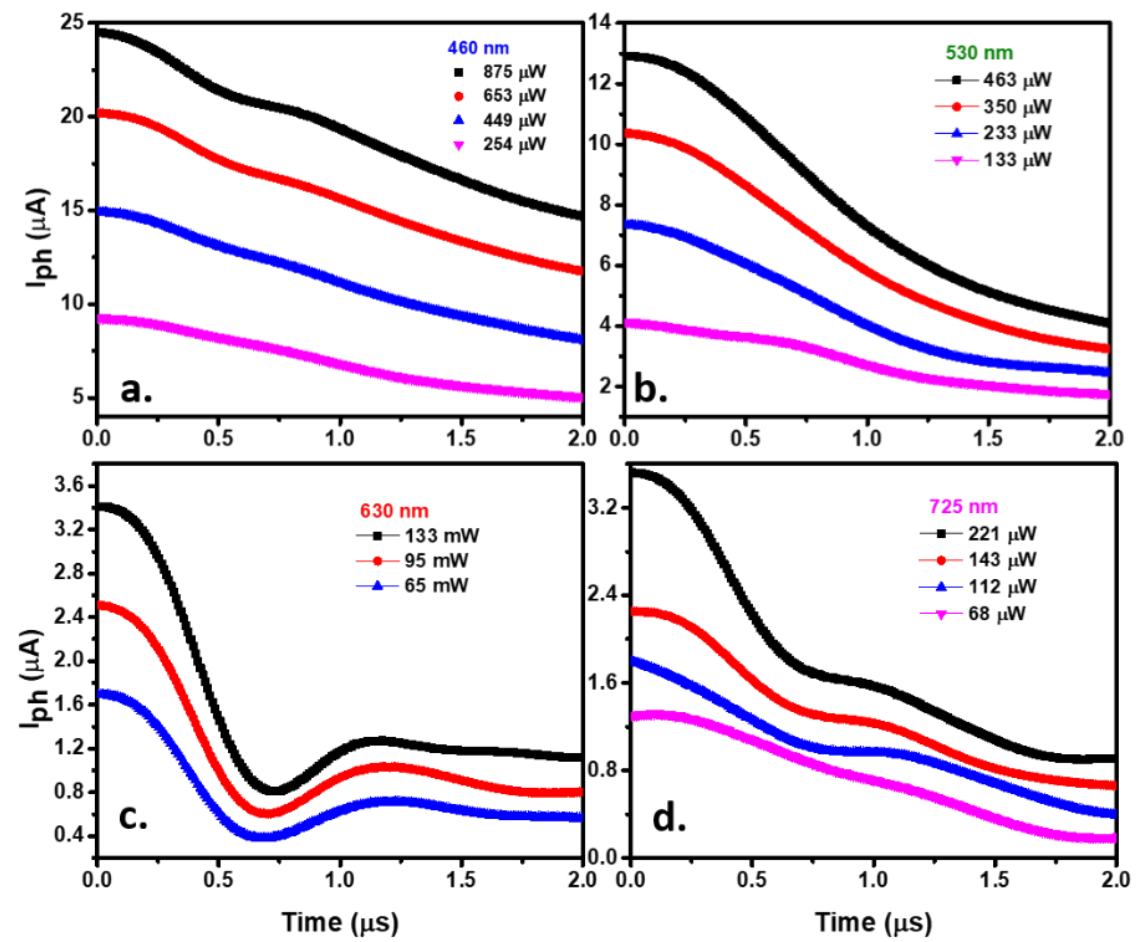

Figure S11. Off transient profiles upon steady state pulsing illumination in a) blue b) green c) red and d) NIR region of the visible spectrum.

12. Circuit analysis of the steady-state photocurrent features:

In a p-i-n perovskite solar cell, the results have been modeled on the basis of two contributions of capacitances; recombination capacitance $\left(\mathrm{C}_{\text {rec }}\right)$ and total/bulk capacitance $\left(\mathrm{C}_{\text {bulk }}\right)$. $\mathrm{C}_{\text {rec }}$ is the equivalent representation of carrier decay due to recombination in a device accompanied by a characteristic time-constant namely the recombination lifetime. $\mathrm{C}_{\text {bulk }}$ is the net capacitance experienced by the photogenerated charge carriers during transport across the bulk (thickness) of the device layer and the interfacial regions. In the case of layered G-HOIP devices, the additional capacitive contribution associated with the organic-linker in between the two quantum well layers can become significant. This barrier capacitance $\left(\mathrm{C}_{\text {barr }}\right)$ acts as accumulation centers for photogenerated carriers.

The simulation results in Figure $\mathbf{S 1 2}$ a, b and c are the current across $\mathrm{R}_{\mathrm{DSO}}(50 \Omega$ input impedance of Digital Signal Oscilloscope, DSO) for variation in $\mathrm{C}_{\text {bulk }}, \mathrm{C}_{\mathrm{rec}}$, and $\mathrm{C}_{\text {barr }}$ respectively. The decay timescales $t_{\text {decay }}, t_{\text {dis }}$ and $t_{r e c}$ which correspond to initial decay, reverse current recovery/discharge time and recombination time respectively are represented in Figure 
4b. As shown Figure S12a, the initial decay timescale $t_{\text {decay }}$ increases linearly with $\mathrm{C}_{\text {bulk. The }}$ same trend is observed in the case of $t_{r e c}$ (Figure S13b) and $t_{d i s}$ (Figure S13c) which varies linearly with $\mathrm{C}_{\text {rec }}$ and $\mathrm{C}_{\text {barr }}$ respectively. As seen in Figure $4 \mathrm{~d}$, the $\mathrm{I}_{\mathrm{ph}}$ decay consists of fast $\left(\mathrm{t}_{\text {decay }}\right)$ and slow decay $\left(\mathrm{t}_{\mathrm{rec}}\right)$ components and can be represented as:

$$
\mathrm{I}_{1}(\mathrm{t})=a \exp \left(-\mathrm{t} / \mathrm{t}_{\text {decay }}\right)+b \exp \left(-\mathrm{t} / \mathrm{t}_{\mathrm{rec}}\right),
$$

(Equation S1)

where $a$ and $b$ are constants. In the case of off-cycle features (Figure 4e), an additional component for the reverse discharge becomes significant. The initial decay component remains while the long-lived recombination parameter need not be considered. The capacitive equation with the discharge and decay term then takes the form;

$$
\mathrm{I}_{2}(\mathrm{t})=a \exp \left(-\mathrm{t} / \mathrm{t}_{\text {decay }}\right)+c\left(1-\exp \left(-\mathrm{t} / \mathrm{t}_{\text {dis }}\right)\right)
$$

(Equation S2)

The values of decay time constants obtained after fitting shows a proportional variation of $t_{\text {decay }}$ and $t_{\text {dis. }}$ The fast decay rates are accompanied by the reverse current discharge at charge accumulation centers.

In case of steady state measurements using periodic light-source, the features in the decay part of $\mathrm{I}_{\mathrm{ph}}(\mathrm{t})$ corresponding to the off-cycle, reveals $\lambda$-dependent characteristics and indicates effects from charging and discharging processes. This method offers an alternative procedure for color sensing where the observed features are cumulative effects of dominant resistive pathways accompanied by capacitive network. The circuit simulation can capture the observed $\mathrm{I}_{\mathrm{ph}}$ features.

The obtained off-cycle decay features obtained in Figure 4e were fit to Equation S2 (fit given in Figure S15b) to obtain the associated decay time scales given in Table S1. Detailed characterization of microstructure and the associated dynamics of the physical processes involved are needed to understand the exact nature of the $\lambda$-dependent trends. It is noted that these features (Figure 4e) are maintained over a range of intensity studied (Figure S11).
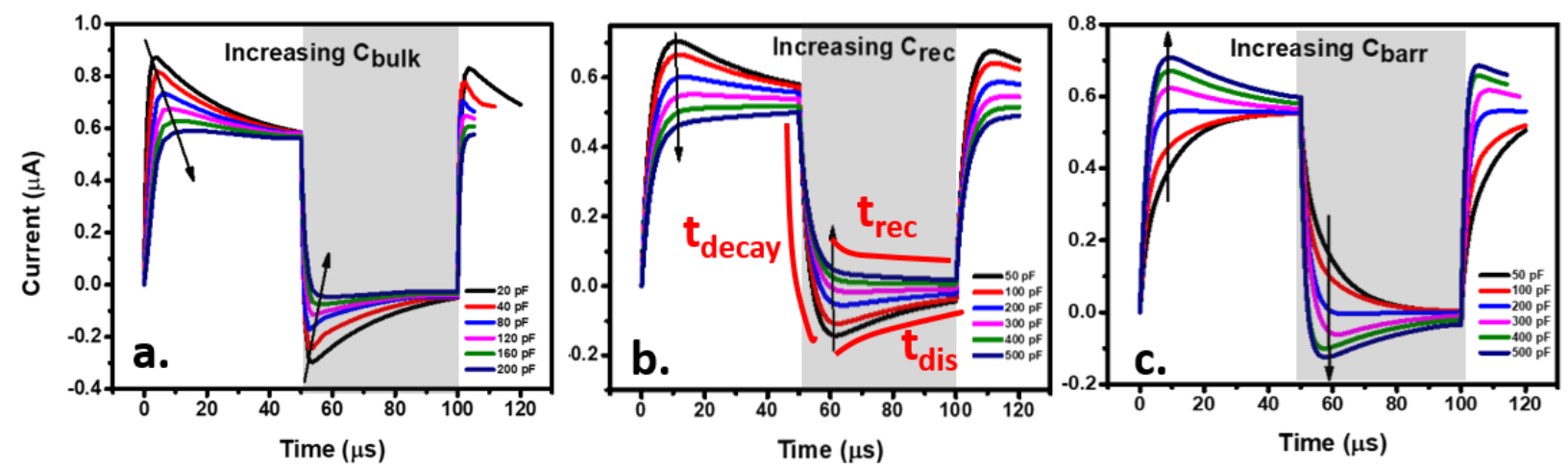

Figure S12. a) Simulated current profiles for light pulsing operation measured across digital signal analyzer $\left(\mathrm{R}_{\mathrm{DSO}}\right)$ in Figure $4 \mathrm{~d}$ for varying $\left.\mathrm{C}_{\text {bulk }}, \mathrm{b}\right) \mathrm{C}_{\mathrm{rec}}$ and c) $\mathrm{C}_{\text {barr. }}$. 
13. Effect of capacitance variation on off-cycle $I_{p h}$ decay features.
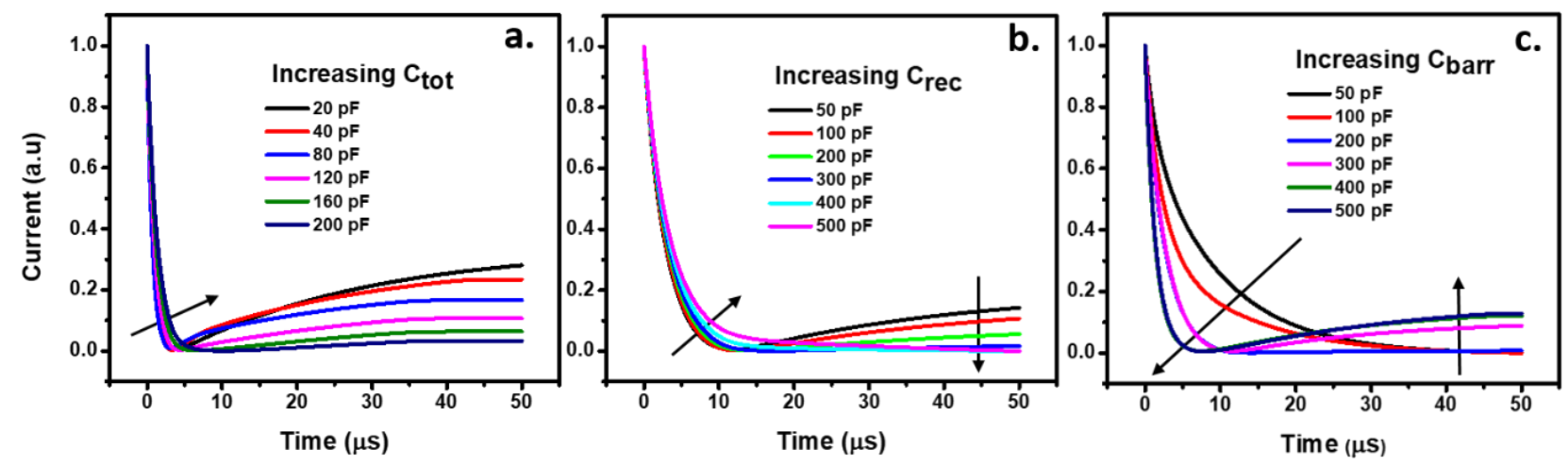

Figure S13. Normalized decay profiles of off-cycle features in Figure S12, indicating effect of capacitance variation: a) $\mathrm{C}_{\text {tot }}$ b) $\mathrm{C}_{\mathrm{rec}}$ and c) $\mathrm{C}_{\text {barr }}$ on decay timescales.

14. Steady-state light pulsing in symmetric hole-only devices and the equivalent circuit.

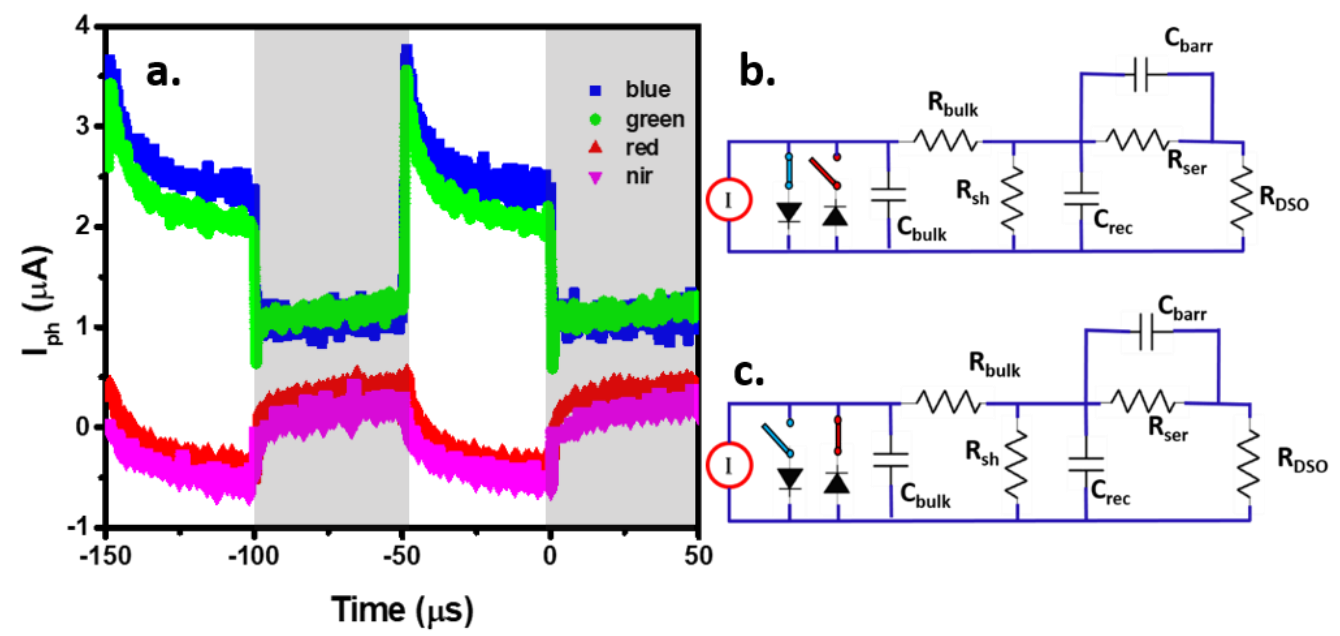

Figure S14. a) Short-circuit $\mathrm{I}_{\mathrm{ph}}$ in symmetric hole-only devices upon light pulsing for different $\lambda$. b) Equivalent circuit for symmetric hole-only device shows 2-diode configuration for both short $\lambda$ and c) long- $\lambda$ illumination. 
15. Fits of capacitive decay equation to transient-OFF features in asymmetric devices.
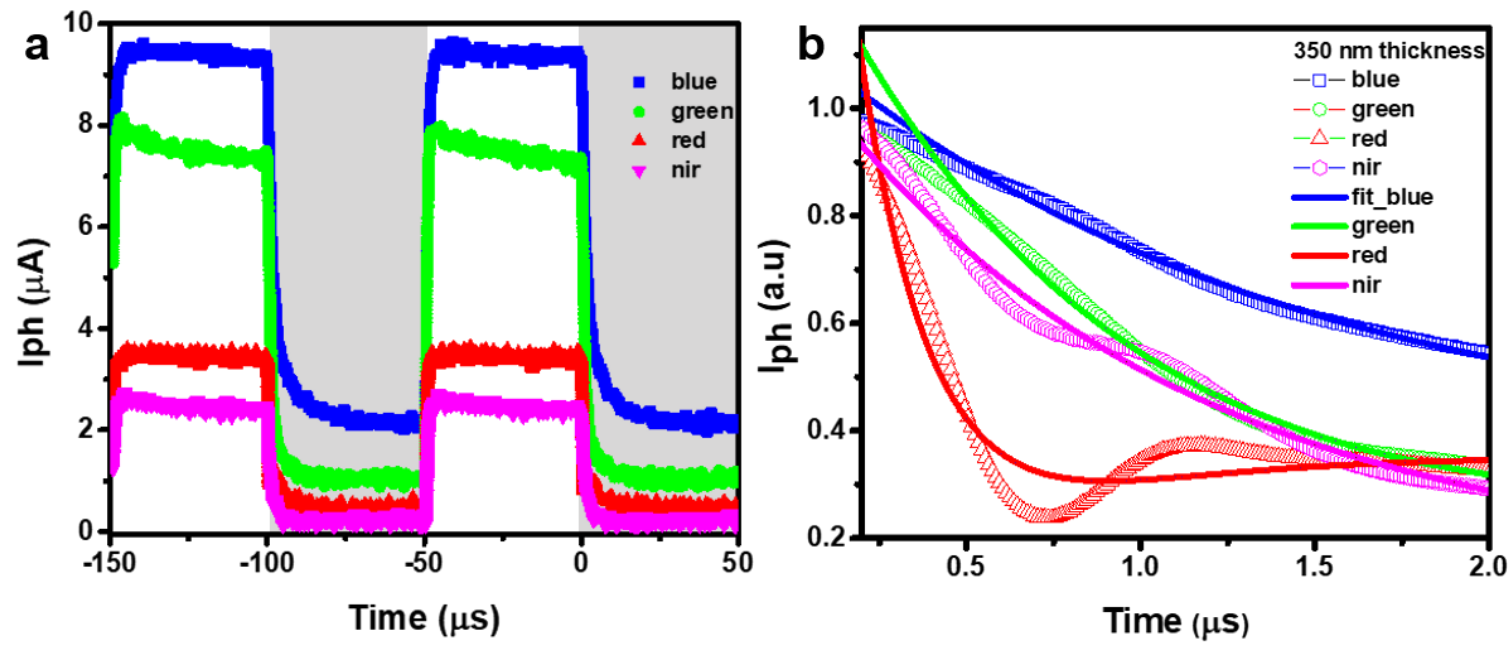

Figure S15. a) Short-circuit $I_{p h}$ in asymmetric devices (active layer $350 \mathrm{~nm}$ thick) upon light pulsing for different $\lambda$ b) Off-cycle shows $\lambda$-dependent features and fits of these features to equation $\mathrm{I}_{2}(\mathrm{t})$.

16. Variation of decay time parameters in off-cycle features for different illumination $\lambda$

\begin{tabular}{|l|l|l|l|}
\hline Color $(\boldsymbol{\lambda})$ & $\mathrm{t}_{\text {decay }}(\boldsymbol{\mu S})$ & $\mathbf{t}_{\text {dis }}(\boldsymbol{\mu S})$ & $\mathrm{t}_{\text {rec }}(\boldsymbol{\mu s})$ \\
\hline Blue $(\mathbf{4 6 0} \mathbf{~} \mathbf{m})$ & 1.4 & 8 & 7.2 \\
\hline Green $(\mathbf{5 3 0} \mathbf{~ n m})$ & 0.98 & 1.97 & 6.8 \\
\hline Red $(\mathbf{6 3 0} \mathbf{~ n m})$ & 0.27 & 0.31 & 4.98 \\
\hline NIR $(\mathbf{7 2 5} \mathbf{~ n m})$ & 0.97 & 0.95 & 2.24 \\
\hline
\end{tabular}

Table S1. Decay time parameters obtained after fitting the off-cycle features (Figure S15) for different $\lambda$ to Equation $\mathbf{S 2}$. 


\section{Experimental Section}

Preparation of graded perovskite films: A $0.3 \mathrm{M}$ perovskite precursor was prepared by adding Butylammonium Iodide (BAI, Sigma Aldrich), Methylammonium Iodide (MAI, Dyesol) and Lead (II) Iodide $\left(\mathrm{PbI}_{2}\right.$, Sigma Aldrich) in the molar ratio 2:4:5 respectively in $\mathrm{N}, \mathrm{N}$ Dimethylformamide (DMF, Alfa Aesar). The films were prepared by the hot-cast method. The glass substrates were first sonicated in Extran soap solution after which it was progressively cleaned with DI water, Acetone and IPA. These substrates were treated with RCA solution which consisted of solution in volumetric ratios of $6: 1: 1$ of DI water, Ammonia solution (EMSURE) and Hydrogen Peroxide (SDFCL) respectively at $120^{\circ} \mathrm{C}$ for $20 \mathrm{~min}$. This was followed by plasma treatment for $5 \mathrm{~min}$. The substrates were then transferred to the glove box to spin coat perovskite precursor under inert conditions. The substrates were first heated up to $150^{\circ} \mathrm{C}$ for $10 \mathrm{~min}$ and then placed on the spin coater chuck onto which the hot perovskite precursor solution $\left(60^{\circ} \mathrm{C}\right)$ was dispensed immediately $(\sim 5 \mathrm{~s})$ and spinned at $5000 \mathrm{rpm}$ for $60 \mathrm{~s}$.

Device Fabrication: Using a $0.3 \mathrm{M}$ precursor solution, the graded bandgap devices were prepared using the hot cast method. The patterned ITO coated glass substrates were sonicated in Extran soap solution and progressively cleaned with DI water, IPA and Acetone. The cleaned substrates were RCA treated at $120^{\circ} \mathrm{C}$ in a solution with volumetric ratios of $6: 1: 1$ of DI water, Ammonia and hydrogen peroxide respectively followed by plasma treatment for $2 \mathrm{~min}$. PEDOT-PSS (Ossila, PEDOT:PSS, AL 4083) solution was filtered using a $0.22 \mu \mathrm{m}$ pore filter and spin coated onto ITO coated substrates at $4000 \mathrm{rpm}$ for $60 \mathrm{~s}$ followed by annealing at $120^{\circ} \mathrm{C}$ for $30 \mathrm{~min}$. The ITO/PEDOT-PSS substrates were transferred to glovebox to spin coat the perovskite precursor under inert conditions. These substrates were heated for $10 \mathrm{~min}$ at $150^{\circ} \mathrm{C}$ 
and transferred to the spin-coater chuck onto which the hot precursor solution was dispensed immediately $(\sim 5 \mathrm{~s})$ and spin coated at $5000 \mathrm{rpm}$ for $60 \mathrm{~s}$.

For the fabrication of asymmetric electrode devices, PCBM (Lumtec Corp.) was then spin-coated at $2000 \mathrm{rpm}$ for $60 \mathrm{~s}$ from a $25 \mathrm{mg} / \mathrm{ml}$ solution in chlorobenzene (anhydrous, Sigma Aldrich). After annealing for $3 \mathrm{~min}$ at $100^{\circ} \mathrm{C}, 100 \mathrm{~nm}$ of $\mathrm{Ag}$ was thermally evaporated using shadow masks to form the top electrode.

In the case symmetric electrode devices, rr-P3HT (Lumtec Corp.) was spin-coated on the ITO/PEDOT-PSS/perovskite films at $2000 \mathrm{rpm}$ for $60 \mathrm{~s}$ from a $14 \mathrm{mg} / \mathrm{ml}$ solution in chlorobenzene (anhydrous, Sigma Aldrich). After annealing for 3 min at $140^{\circ} \mathrm{C}$, both $\mathrm{MoO}_{\mathrm{x}}(9$ $\mathrm{nm})$ and $\mathrm{Ag}(100 \mathrm{~nm})$ were thermally evaporated using shadow masks to form the top electrode.

All the devices were encapsulated using UV-curable epoxy to reduce effects of air and moisture induced degradation.

\begin{abstract}
Absorption Spectroscopy: Thin film absorption spectroscopy was performed using a UV/Vis/NIR spectrometer (PerkinElmer Lambda 750) in the transmission geometry. Graded perovskites spin coated on glass slides were excited in the spectral range of 400-850 $\mathrm{nm}$ and the absorption measured simultaneously.
\end{abstract}

PL spectroscopy: PL spectroscopy was performed on graded perovskite thin films. A $470 \mathrm{~nm}$ excitation laser source (PicoQuant GmbH LDH-P-C-470), operated using a laser driver(PicoQuant GmbH PDL 800-B), was incident on the sample and the reflected light after passing through a $500 \mathrm{~nm}$ long pass filter was collected using a fiber coupled Mini Spectrometer (Hamamatsu, C10083CA). The perovskite thin film coated on glass slide was flipped to record the PL spectrum for light reflected from either sides of the sample. 
Photoluminescence Mapping: Ziess-700 confocal microscope is used to acquire the confocal images. A $488 \mathrm{~nm}$ laser diode was utilized to excite the sample. The emission is collected using two different channels (PMTs) operating simultaneously. One of the channel had long-pass 640 $\mathrm{nm}$ filter (red color used during image reconstruction) and other had short-pass $640 \mathrm{~nm}$ filter (green color used during image reconstruction). The two channel measurements were then overlaid to construct the confocal maps. The pinhole used is 1 Airy unit.

Transient Absorption spectroscopy: The fundamental output of a Ti:Sapphire amplifier system (Spectra-Physics Solstice) operating at $1 \mathrm{KHz}$ (Pulse wavelength $=800 \mathrm{~nm}$, pulse width = $90 \mathrm{fs}$ ) was split into two beams. One beam pulse was converted into the broadband visible probe by the home-built noncollinear optical parametric amplifier (NOPA). Another beam was passed through second harmonic generation (SHG) beta barium borate crystal to generate the $400 \mathrm{~nm}$ pump pulse. $400 \mathrm{~nm}$ pump was used to excite the sample and visible broadband pulse is probed to observe the photoinduced absorption changes as a function of time. The pump and probe pulses are both incident from the same side of the sample. The delay between the pump and probe was obtained by the motorized translation stage. The pump and probe beams are overlapped on to the sample from the same side. The transmitted probe beam was collected with an InGaAs dual-line array detector (Hamamatsu G11608-512). The transmission difference was read out by a custom-built lock-in board from Stresing Entwicklungsbüro.

Transient Photocurrent: The fundamental output of a Ti:Sapphire amplifier system (SpectraPhysics Solstice) operating at $1 \mathrm{KHz}$ (Pulse wavelength $=800 \mathrm{~nm}$, pulse width $=90 \mathrm{fs}$ ) was passed through the commercial optical parametric amplifier TOPAS (Light Conversion). The monochromatic light pulses $(<10 \mathrm{~nm})$ from the TOPAS is incident on the device from the ITO 
side. The short-circuit photocurrent is measured across the $50 \mathrm{ohm}$ resistor in series with the device. The data is acquired using Agilent Infiniium (DSO80304B, $40 \mathrm{GSa} / \mathrm{s}$ ) oscilloscope.

Steady State pulsing measurements: Steady state measurements were performed on graded perovskite devices using commercially available LEDs. The LED was modulated using a function generator (Tektronix), and the same input was used as a trigger to sample the data from the graded perovskite device. The light was focused on the device area using an optical lens and the current was amplified using a pre-amplifier (Femto HCA-100M-50K-C, 50KV/A, 100 $\mathrm{MHz}$ ) before being input into the oscilloscope (LeCroy waveRunner 6100A, $1 \mathrm{GHz}$ ).

\section{Acknowledgements}

We acknowledge Dr. Balaji Jayaprakash, Center for Neuroscience, Indian Institute of Science, Bengaluru for providing with the 2-photon microscopy images. 